\title{
Identifying Artifacts in Arterial Blood Pressure Using Morphogram Variability
}

\author{
W Ali ${ }^{1}$, L Eshelman', M Saeed ${ }^{2}$ \\ 'Philips Research USA, Briarcliff Manor, NY, USA \\ ${ }^{2}$ Philips Monitoring Division, Andover, MA, USA
}

\begin{abstract}
In this article we present a simple technique that utilizes the cross correlations between ECG signals and haemodynamic signals for the purpose of assessing signal quality and detecting artifacts in the arterial blood pressure (ABP) signal. The technique was tested using cases from a physician-annotated patient monitoring signal database from Beth Israel/Harvard-MIT University data bank. The results were encouraging: $90 \%$ of the manually annotated artifacts were correctly classified as artifacts and $99 \%$ of the manually annotated true events were correctly classified (out of a total of 683 manually annotated alarms).
\end{abstract}

\section{Introduction}

Alarms due to artifacts in monitoring signals reduce the efficacy of healthcare provision, especially in intensive and critical care units (ICU/CCU). Thus, it is important to develop a method for identifying these false alarms (based on artifacts) versus actual alarms (due to real changes in a patient's underlying physiologic state).

Numerous systems have been developed for automatically analyzing patient monitoring signals. These systems have employed various methods ranging from traditional signal processing techniques such as frequency analysis, time-frequency analysis, and wavelet analysis [5][3][7] to techniques developed by Artificial Intelligence researchers [4][1]. Most of these systems use a single-signal model, or when multiple channels are analyzed, they are of the same type of signal - such as ECG signals from multiple leads. However, there are a several important exceptions Error! Reference source not found.[8][9]. Tsien looked at a number of patient monitoring. signals, including blood pressure, carbon dioxide, oxygen and heart rate, exploring a number of machine learning techniques for identifying artifacts and comparing single channel and multi-channel approaches. Zong, et al. developed a system for detecting artifacts in arterial blood pressure (ABP) signals by analyzing the relationship between the ABP signal and the ECG signals using a fuzzy logic approach to evaluate signal quality of the ABP waveform [9]. Both of these studies found that the multi-signal approach was more effective than simply analyzing the targeted signal. Although much could be said about the strengths and weaknesses of these various methods, the algorithm complexity was quite significant.

In the next section we shall describe a simple method that requires minimal computational power for exploiting the relationships among signals used in patient monitoring. In the third section we shall present experimental results that demonstrate the effectiveness of this method for detecting artifacts in ABP signals, and thereby reduce false blood pressure alarms.

\section{Methods}

The fundamental premise of this study is that relationships among certain patient monitoring signals can be used to assess a particular signal (e.g., whether it is an artifact) in light of the behavior of other signals. Here we will be relying on the correlation between ECG and ABP signals, using the ECG for examining the ABP's fidelity. The proposed method represents the interaction of the monitored signals as morphograms and specifies rules for interpreting changes to these morpograms. We use the term "morphogram" to describe the plotting of one signal versus another for a given period of time -e.g., an ECG signal on the $x$-axis and the ABP on the $y$ axis.

We investigated if the $A B P$ and the various ECG signals are highly correlated by determining if a characteristic morphology or signature is present. The signature represents the correlated signals for a single heart beat. Since the time period used is greater than one heart beat, significant departures from this signature can be seen on the morphogram plots. These departures indicate either a physiologically caused event or an artifact. The underlying heuristic of the morphogram algorithm is that physiologically caused events are more likely to affect all signals, and thus there will be perturbations in all morphograms, whereas artifacts are more likely to affect only a single signal, and thus there will be perturbations only in the morphograms involving that signal. Figure 1 shows a 12 second plot of a typical set of signals being monitored for a patient with 
respiratory failure [6]. Figure 3 shows the three morphograms created using two of the ECG signals (II and $V$ ) and the ABP signal. Each morphogram subplot has a well-defined shape that repeats itself with time as long as there are no artifacts and the patient is not undergoing any physiological changes. Not only do the morphograms for each patient have a well-defined shape, but often the signatures of the two ABP-ECG morphograms and the ECG-ECG morphogram have characteristic shapes that can be generalized across patients.

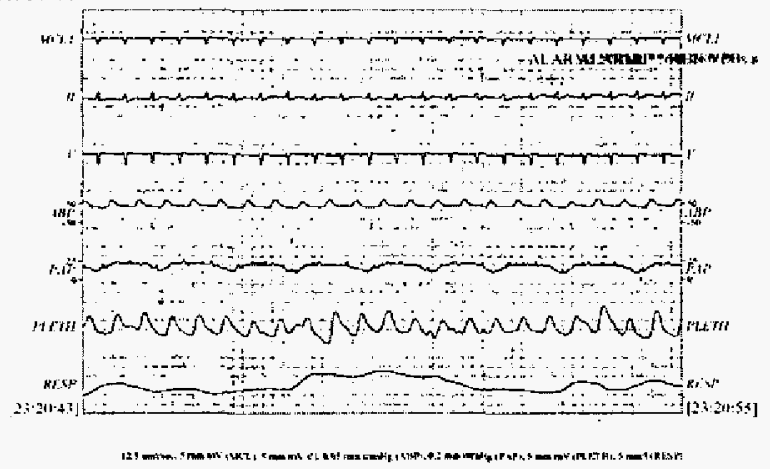

Figure 1 MCL1, II, and V ECG leads, ABP, PAP, PLETH, and RESP monitoring signals
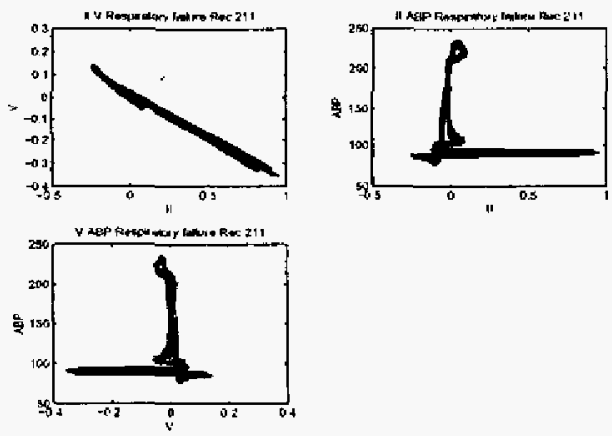

Figure 2 Morphograms of II, V, and ABP signals

Since the ABP wave lags behind the QRS complex, the ABP-ECG morphograms will typically have the characteristic "L" shape (or backwards "L" shape). Assuming that the ABP value is mapped onto the $Y$-axis and the ECG value is mapped onto the $\mathrm{X}$-axis, then when the $A B P$ is at a low $Y$ value, the ECG is approaching its maximum (or minimum), and similarly when the ECG is approaching its neutral value, the ABP is approaching its maximum. This is what accounts for the characteristic " $L$ " shape of the ABP-ECG morphograms. On the other hand, the ECG-ECG morphograms trace out a pattern that lies along the diagonal, either at 45 degrees or 135 degrees (usually as a bar or an oval). This is because both signals reach their extremes (some point in the QRS complex) in unison.

Any change in the pattern of a signal will be quite obvious in the morphograms using that signal, i.e., there will be a perturbation of each morphogram's signature morphology. Given that the signals are highly correlated, a change in all the morphograms probably indicates a physiologically caused event, whereas a change in the subset of the morphograms that have a single signal in common probably indicates an artifact (e.g., a mechanical problem for that signal). Figure 3 shows an example where all the morphograms (for the same patient) are perturbed because of physiological changes in the patient's condition.
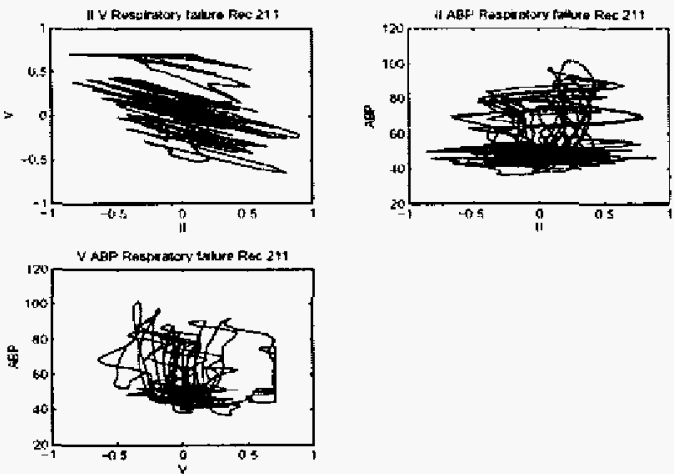

\section{Figure 3 Perturbations in all the morphograms}

Figure 4 , on the other hand, shows the morphograms for a patient with pulmonary edema during a time period in which an artifact occurs in the ABP signal. Note that only the morphograms that include the ABP signal are perturbed. The morphogram for the two ECG signals retains its characteristic diagonal-oval shape, whereas the two morphograms in which the ABP signal is plotted against either of the two ECG signals are highly distorted.
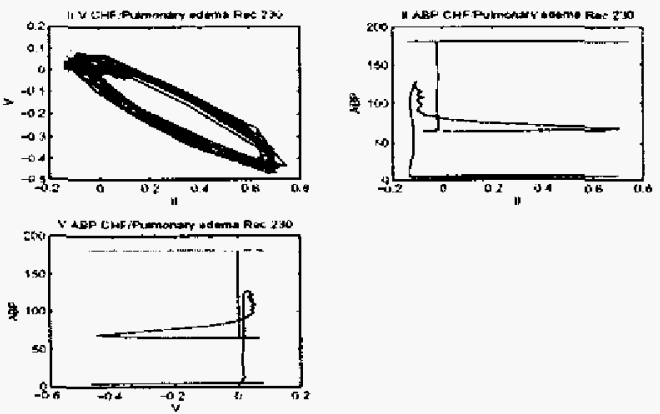

Figure 4 Non-consistent perturbations in the different morphograms indicating an artifact

This is a clear indication of an artifact presence in the 
signal that is common to the second and third morphogram subplots, namely, the ABP signal. In order to exploit morphograms we need to describe how perturbations are determined. Ideally one could determine the "normal" morphogram signature, with limits, for a particular patient, and then detect when the pair of signals being traced significantly exceeds these limits. For example, the algorithm could determine the convex hull for the morphogram. However, this is computationally expensive, and one of our goals has been to try to keep the algorithm as simple as possible. Our method is to enclose the morphogram in a rectangle whose sides are parallel to the $x$ and $y$ axes, and then calculate the area of this rectangle. The algorithm divides the 15 -second period preceding the alarm into equal one-second, nonoverlapping segments and creates a morphogram for each segment. Rectangular areas are computed for each segment. The minimum and maximum rectangular areas for these segments are identified. Finally, the ratio of the difference between the maximum and minimum areas to the minimum area is computed and compared to a threshold. We interpret the alarm as an artifact if the ratio for the ECG pair of signals is below the first threshold and both ABP ratios (one for each ECG lead) are above a second threshold:

(ratio_of_rect_areas(ecg1,ecg2)<threshold_ecg) d

(ratio_of_rectr_areas $(a b p, e c g I)>$ threshold_abp) \&

(ratio_of_rect_areas(abp,ecg2) > threshold_abp)

where ratio_of_rect_areas $(s 1, s 2)$ is:

(max_rect_area - min_rect_area) / min_rect_area

The following examples (for a patient with respiratory failure) illustrate how the algorithm works, using the two ECG leads (II and V) and the ABP signal. Figure 5 shows the signals being monitored during the period of an alarm that has a physiological cause. Figure 6 shows the three morphogram subplots. Each subplot includes the 15 second morphogram (in blue) and the fifteen encapsulating rectangles (in red). (For purposes of easy visualization, the rectangles have been shifted so that their lower left hand corners lie at the same point.) In a case where at least one signal is unstable there will be a lot of variability in the areas of the rectangles. There are significant changes in all the morphograms, indicating the presence of changes not only in the ABP signal, but also in the ECG signals over the period of study. In the second example the alarm is caused by an artifact (Figures 7 and 8 ). In the morphograms there are significant changes in only two of the morphogram subplots - the $2^{\text {nd }}$ and $3^{\text {rd }}$, which are the ones involving the ABP. It should be noted that the morphogram heuristic has an inherent weaknesses---if there is an ABP artifact at the same time that there is significant noise in the ECG signal, then the algorithm will interpret the alarm as having a physiological cause. In order to deal with this problem, two additional rules were added to the morphogram to identify fairly obvious cases of ABP artifacts. This leaves the more subtle cases to the morphogram rule. The first rule classifies the ABP signal as an artifact whenever there is a stretch of more than $L$ consecutive sample values that are equal due to saturation. The second rule classifies the ABP signal as an artifact whenever the ABP signal range has narrowed for a sustained period of time due to overdamping.

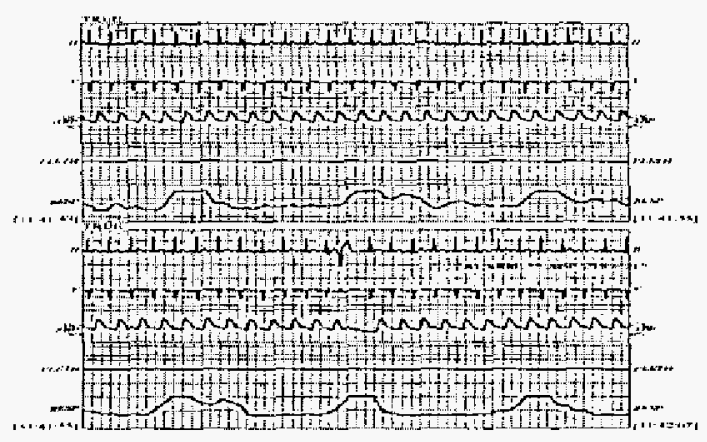

Figure 5 Monitored signals for the period starting 20 seconds before and ending 4 seconds after the alarm
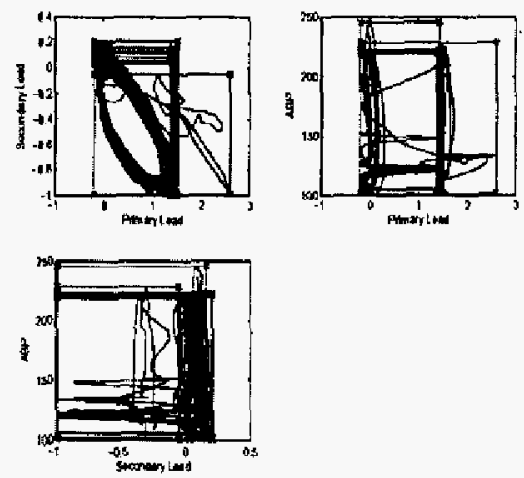

Figure 6 Morphograms for a physiologically caused ABP-based alarm

\section{Results}

We tested the morphogram algorithm on the publicly available MIMIC-I (Beth-Israel Hospital) data base [6] using the artifact annotations provided by Zong [9]. We used the following parameter settings for the three rules: the morphogram rule with ECG and ABP threshold settings of 0.8 and 1.7 , respectively, the rule for detecting saturation with a threshold of 15 , and the rule for 
detecting overdamping with thresholds of $8 \mathrm{mmHg}$ and 250 samples. (The results were similar for a wide range of parameters we tested, indicating that the algorithm is quite robust.). Out of 160 manually annotated artifacts, the morphogram algorithm detected 144 (as artifacts) and missed 16 (i.e., classified them as true alarms whereas they had been manually annotated as artifacts). Out of 523 manually annotated true alarms, the algorithm classified 518 as true alarms and 5 mistakenly as artifacts. Thus, for this experiment the sensitivity was $90 \%$ and the specificity was $99 \%$.

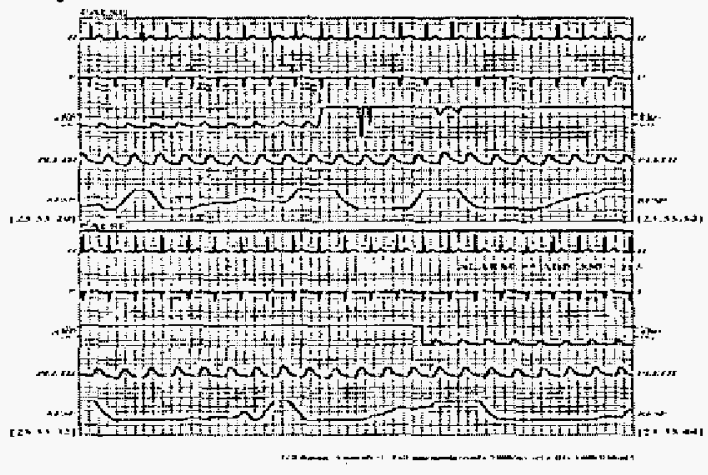

Figure 7 Monitored signals for the period starting 20 seconds before and ending 4 seconds after the alarm
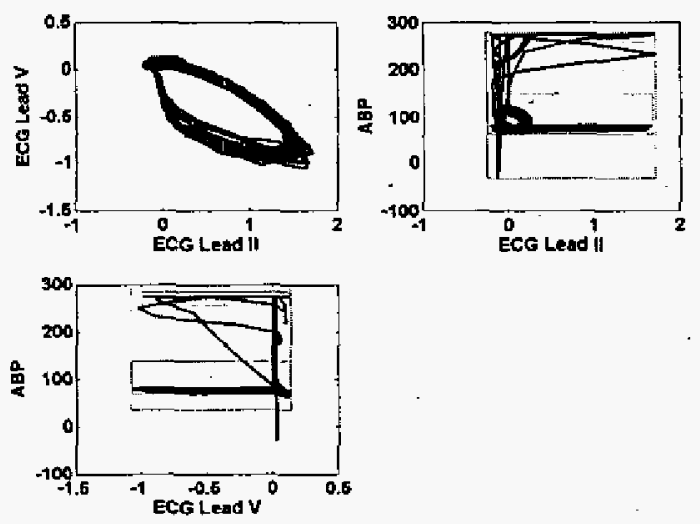

Figure 8 Morphograms for an ABP-based alarm caused by an artifact

\section{Discussion and conclusions}

In this paper we have presented an algorithm that detects artifacts in ABP signals via morphograms, where abnormalities in the signals show up as perturbations in morphogram shapes. A simple method was developed for classifying these perturbations as either caused by clinical conditions or by artifacts and was tested for detecting ABP artifacts using a physician-annotated database with encouraging results. Finally, it should be stressed that the morphogram algorithm is not limited to detecting artifacts in ABP signals, but can easily be applied to other signals.

\section{Acknowledgements}

The authors are very grateful for the support and suggestions made by Larry Nielsen from Philips monitoring division in Andover and Professor Roger Mark of MIT for many useful discussions.

\section{References}

[1] Cabello, D.; Barro, S.; Ruiz, R.; Zapata, E.L.; Mira, J.; "Fuzzy clustering application to the diagnosis of ventricular arthythmias" Engineering in Medicine and Biology Society, 1988. Proceedings of the Annual International Conference of the IEEE , 4-7 Nov. 1988, Page(s): $5-6$ vol. 1

[2] Crew A.D., Stoodley K.D.C, Lu R., Old S., and Ward M.; "Preliminary clinical trials of a computer-based cardiac arrest alarm". Intensive Care Medicine 1991; 17:359-364

[3] Cuiwei $\mathrm{Li}$; Chongxun Zheng; Changfeng Tai; "Detection of ECG characteristic points using wavelet transforms" Biomedical Engineering, IEEE Transactions on , Volume: 42 Issue: 1 , Jan. 1995, Page(s): 21 -28

[4] Frenkel, D.; Nadal, J.; "Ischemic episode detection using an artificial neural network trained with isolated ST-T segments" Computers in Cardiology 1999 , 26-29 Sept. 1999, Page(s): $53-56$

[5] Hamilton, P.S.; Tompkins, W.J.; "Adaptive matched filtering for QRS detection" Engineering in Medicine and Biology Society, 1988. Proceedings of the Annual International Conference of the lEEE , 4-7 Nov. 1988 , Page(s): $147-148$ vol.1

[6] hutp://www.physionet.org

[7] Reyna, M.A.; Jane, R.; "Detection of ventricular tachycardia risk by means of Wigner Distribution Interference Terms from the high resolution ECG" Computers in Cardiology $1997,7-10$ Sept. 1997, Page(s): $625-628$

[8] Tsien C.L. and Kohane I.S.; "Using multi-signal integration to detect false alarms in intensive care unit". Medical Decision Making 1998; 18(4):469.

[9] Zong W, Moody G.B., and Mark R.G. "Reduction of False BP Alarms by Use of ECG-BP Relationships". hitp://www.phvsiontet.org

Address for correspondence

Mohammed Saeed

45 Carleton St. E25-505

Cambridge, MA 02142

msaeed@mit.edu 